\title{
A Moving Target Tracking Control of Quadrotor UAV Based on Passive Control and Super-Twisting Sliding Mode Control
}

\author{
Wentao Xue $\left(\mathbb{D}\right.$, Xunnan Zhu, Xiaofei Yang $\mathbb{D}^{\mathbb{D}}$, Hui Ye $\mathbb{D}$, and Xuan Chen \\ School of Electronic and Information, Jiangsu University of Science and Technology, Zhenjiang 212100, China \\ Correspondence should be addressed to Xiaofei Yang; yxfei_0809@just.edu.cn
}

Received 18 December 2020; Accepted 2 May 2021; Published 11 May 2021

Academic Editor: Gang Lei

Copyright ( 2021 Wentao Xue et al. This is an open access article distributed under the Creative Commons Attribution License, which permits unrestricted use, distribution, and reproduction in any medium, provided the original work is properly cited.

\begin{abstract}
A novel asymptotic tracking controller for an underactuated quadrotor unmanned aerial vehicle (UAV) is proposed to solve a moving target tracking problem. Firstly, the control system is decoupled into the position control system and the attitude control system. Secondly, a method combined artificial potential field with passivity control (APF\&PC) is introduced for the positioning system to achieve high-precision tracking of moving target at a fixed distance. Thirdly, a super-twisting sliding mode (STSM) method with an improved reaching law for the attitude system is applied to ensure that the attitude converges to the desired value. Furthermore, the stabilities of two subsystems are proved, and sufficient stability conditions are derived based on the passive method and Lyapunov method, respectively. Finally, simulation results of the moving target tracking verify the superiority and robustness of the proposed control method in the presence of parameter uncertainties and external disturbances.
\end{abstract}

\section{Introduction}

In recent years, quadrotor unmanned aerial vehicles have attracted increasing attention from both industrial and academic communities. With some characteristics of vertical take-off and landing, single or team flight, and low-cost manufacturing, they have wide applications such as military monitoring, traffic information collection, power system detection, and disaster first aid [1-4]. The quadrotor UAV is an underactuated and strongly coupled nonlinear system, which is subject to structural uncertainties and unknown external disturbance. Therefore, it is a great challenge to design an accurate and robust controller for the quadrotor to achieve autonomous flight and target tracking.

Moving target tracking is an essential skill for the quadrotor UAV to perform military and civil tasks, like tracking enemy targets in air combat, tracking vehicles in urban antiterrorism, and timely finding and locking the victims in maritime rescue $[5,6]$. Due to the limitation of the observation range and the danger of unfriendly targets, the quadrotor UAV needs to maintain a certain distance in the whole mission, which brings new challenges to target tracking. Currently, many kinds of control methods have been successfully applied in the target tracking of UAVs. Wang et al. [7] proposed an integrated framework for UAVbased target tracking and recognition systems to achieve satisfactory tracking performance. Rabah et al. [8] developed a target tracking algorithm based on a Fuzzy-PI controller to track a moving target under varying speed. Araar et al. [9] proposed a PID method with an EKF filter to track autonomously and land on a moving platform. Chen et al. [10] presented sliding mode control methods based on artificial potential field and RBF neural network for a quadrotor to track the moving target. Based on studies aforesaid, the design of a controller with high precision and strong robustness is the precondition of accurate tracking. Thus, it is the key for the moving target tracking of a quadrotor to realize the position and attitude tracking control of the quadrotor system and improve the robustness of the system.

In order to achieve attitude stabilization and position tracking control of the quadrotor, various advanced control techniques have been developed. Many remarkable achievements have been obtained such as robust PID control [11], active disturbance rejection method [12], feedback linearization control [13], backstepping control [14], model predictive control [15], adaptive control [16], immersion and 
invariance control [17], reinforcement learning [18], and composite stability control [19, 20]. Although the above methods can accomplish the autonomous flight of UAVs, some of them still have limited capability to handle uncertainties and external disturbances. Sliding mode control (SMC) is considered an effective method in solving the control problems of uncertain nonlinear systems because of its unique robustness and adaptability to uncertainty and external disturbances [21-23]. Therefore, a wide class of control methods based on SMC has been proposed to solve trajectory tracking of the quadrotor. Chen et al. [24] designed a robust nonlinear controller that combines sliding mode control and backstepping control to achieve trajectory tracking of a quadrotor UAV. Xu et al. [25] presented a quadrotor controller based on the terminal sliding mode control to track the attitude trajectory under input saturation. Castañeda et al. [26] proposed an adaptive second-order sliding mode control method to improve the robustness of the system under different operating conditions and external disturbances. Inspired by the aforementioned papers, we can improve sliding mode control to handle the attitude control problem of quadrotors under uncertainties and external disturbances.

There are some main feasible control methods to realize the position tracking of UAVs. The artificial potential field (APF) is a robust feedback control method based on the virtual force field, which has been widely applied in obstacle avoidance and target tracking [27]. Chen et al. [28] proposed an artificial potential field UAV path planning method to make UAV avoid obstacles efficiently and economically when there are many threats. Hu et al. [29] designed a timevarying sliding surface and a nonlinear adaptive feedback control law based on the APF method for obstacle avoidance control. The passive control (PC) method is to redistribute the energy function of the inverted system by injecting damping into the system so that it conforms to Lyapunov stability law and ensures that the system is asymptotically stable [30]. Ha et al. [31] proposed a unified passivity-based adaptive backstepping control framework for the velocity field following and timed trajectory tracking of a quadrotor on the Internet. Jose and Hugo [32] presented a passivitybased controller for a quadrotor with a cable-suspended load to guarantee asymptotic stability. Based on the aforementioned discussion, we propose a novel double-loop control system based on the position controller and attitude controller, which can achieve high-precision target tracking for the quadrotor. The position controller combines the artificial potential field with passive theory to achieve high-precision position tracking, and the attitude controller uses the supertwisting sliding mode method with an improved reaching law to improve the convergence speed of the attitude.

The main contributions of this study are as follows: firstly, a novel position controller based on artificial potential field and passive control is proposed to improve the precision of fixed distance tracking. The quadrotor can track the moving target at a certain distance by tracking the balance point of the artificial potential field. The passive control can improve the robustness of the system by providing disturbance rejection to uncertainties and disturbances. Besides, a super-twisting sliding mode controller with an improved reaching law is presented for attitude control to ensure fast convergence and strong robustness. The reaching law based on proportional term and exponential term can reduce the chattering and improve the convergence rate, which will guarantee a better tracking performance.

The rest of this paper is organized as follows. In Section 2 , the structure of the target tracking system and the dynamic modeling of the quadrotor are presented. In Section 3, a novel quadrotor control method for moving target tracking is studied and its stability is analyzed. In Section 4, numerical simulations are provided to show the feasibility and effectiveness of the proposed approach. Finally, a brief conclusion is drawn in Section 5.

\section{Mathematical Model of the Quadrotor}

Target tracking is a complex engineering system that involves technical modules in hardware, localization, mapping, planning, and control. The target tracking of UAV is to locate the target according to the distance information of relative observation angle and attitude information of UAV. Then, the future motion state of the target is predicted according to the observation data of sensors. In addition, the target state is filtered and estimated, and motion information of UAV is collected. On the premise that the attitude angle and angular velocity of the quadrotor can be measured, the three-dimensional position and translation velocity of the UAV relative to the moving target are available. Finally, the UAV will be guided to keep track of the moving target through the continuous update of target estimation and prediction state. For the target tracking of the quadrotor, the most critical issues are the control of trajectory tracking. We assume that the relative position of the quadrotor can be obtained from the visual information of the camera, and the state of the tracking target can be estimated by the Kalman filtering method. In this paper, we will focus on the trajectory tracking of the quadrotor control system. By designing the position controller and attitude controller of quadrotor UAV, the moving target can be tracked within a safe distance. The simplified structure of the tracking system is shown in Figure 1.

The dynamical model of the quadrotor is set up by the earth-frame $I(O x y z)$ and the body-frame $B(O x y z)$ as illustrated in Figure 2. It is assumed that the moving target is a mobile car, and $\rho$ is the relative distance between the quadrotor and target. In quadrotor systems, $f_{i}(i=1,2,3,4)$ stands for the thrust force produced by the $i$ th propeller. The vertical motion is created by the collective thrust input of all four propellers. The roll, pitch, and yaw motion can be achieved by changing the speeds of different propellers. The position and attitude of the quadrotor expressed in the inertial frame are defined as $\mathbf{P}=[x, y, z]^{\mathrm{T}}$ and $\boldsymbol{\Theta}=[\phi, \theta, \psi]^{\mathrm{T}}$, where $\phi, \theta$, and $\psi$ are successively roll, pitch, and yaw angles. These angles are bounded as $\phi \in(-\pi / 2 \pi / 2), \theta \in$ $(-\pi / 2 \pi / 2)$, and $\psi \in(-\pi \pi)$.

The dynamic behavior of quadrotor in the inertial coordinate can be described by the dynamic equation based on the Euler-Lagrange method [33] as follows: 


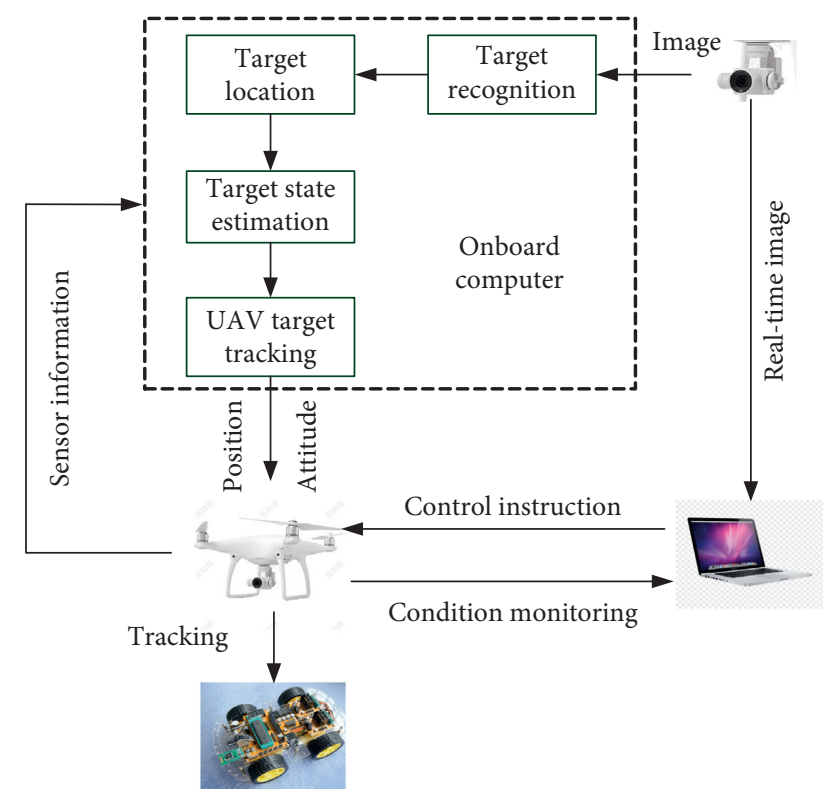

FIGURE 1: Structure diagram of the tracking system.

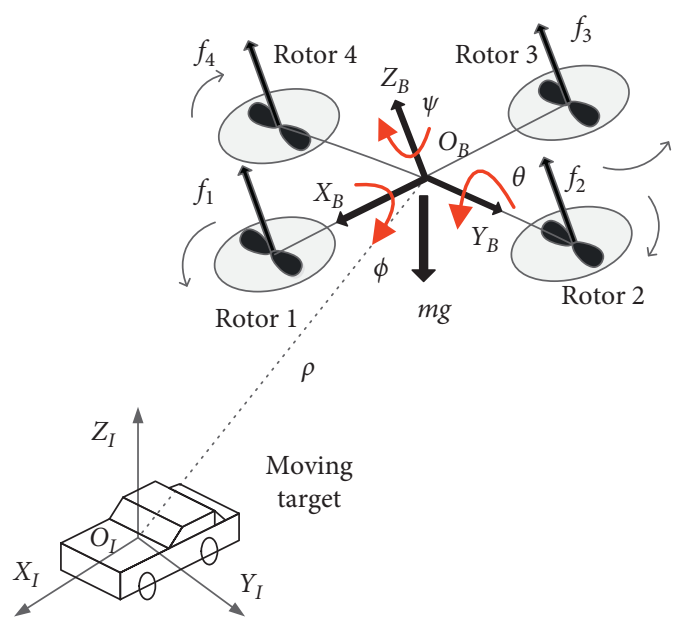

Figure 2: Model diagram of the tracking system.

$$
\begin{aligned}
m \ddot{P} & =U_{1} \mathbf{R} e_{3}-m g \mathbf{e}_{3}+\mathbf{d}_{1}, \\
\mathbf{J} \ddot{\Theta} & =\Gamma-\mathbf{C} \dot{\Theta}+\mathbf{d}_{2},
\end{aligned}
$$

where $m$ is the total mass, $g$ is the gravitational acceleration, and $\mathbf{e}_{3}=\left[\begin{array}{lll}0 & 0 & 1\end{array}\right]^{\mathrm{T}}$ is a unit vector in the vertical direction. The vectors $\mathbf{d}_{1}$ and $\mathbf{d}_{2}$ denote, respectively, the normbounded uncertainties and disturbances in translational and rotational motions, which are slow time-varying signals.

$\mathbf{R}$ represents an orthonormal rotation matrix, defined by

$$
\mathbf{R}=\left[\begin{array}{ccc}
C_{\theta} C_{\psi} & S_{\theta} S_{\phi} C_{\psi}-C_{\phi} S_{\psi} & C_{\phi} S_{\theta} C_{\psi}+S_{\phi} S_{\psi} \\
C_{\theta} S_{\psi} & S_{\phi} S_{\theta} S_{\psi}+C_{\phi} C_{\psi} & C_{\phi} S_{\theta} S_{\psi}-S_{\phi} C_{\psi} \\
-S_{\theta} & S_{\phi} C_{\theta} & C_{\phi} C_{\theta}
\end{array}\right],
$$

where $C_{(.)}$and $S_{(\cdot)}$, respectively, represent cosine function and sinusoidal function.
The positive definite matrix $\mathbf{J}$ is the moment of inertia, given by

$$
\mathbf{J}=\left[\begin{array}{ccc}
I_{x x} & 0 & -I_{x x} S_{\theta} \\
0 & I_{y y} C_{\phi}^{2}+I_{z z} S_{\phi}^{2} & \left(I_{y y}-I_{z z}\right) S_{\phi} C_{\phi} C_{\theta} \\
-I_{x x} S_{\theta} & \left(I_{y y}-I_{z z}\right) S_{\phi} C_{\phi} C_{\theta} & I_{x x} S_{\theta}^{2}+I_{y y} S_{\phi}^{2} C_{\theta}^{2}+I_{z z} C_{\phi}^{2} C_{\theta}^{2}
\end{array}\right],
$$

where $\mathbf{I}=\left[\begin{array}{lll}I_{x x} & I_{y y} & I_{z z}\end{array}\right]^{\mathrm{T}}$ represents inertia tensors of the quadrotor.

The vector $\mathbf{C}$ represents a Coriolis and centrifugal force term, which can be calculated by [33]

$$
\mathbf{C}=\dot{J}-\frac{1}{2} \frac{\partial}{\partial \Theta}\left(\dot{\Theta}^{T} \mathbf{J}\right) \text {. }
$$

The control input $U_{1}$ denotes total lift force and $\Gamma=$ $\left[\begin{array}{lll}\Gamma_{\phi} & \Gamma_{\theta} & \Gamma_{\psi}\end{array}\right]^{\mathrm{T}}$ stand for torques applied on the three axes, which can be calculated by rotor thrusts as follows [34]:

$$
U_{1}=\sum_{i=1}^{4} f_{i}=\sum_{i=1}^{4} b_{1} \omega_{i}^{2}
$$

where $b_{1}$ and $\omega_{i}$ represent lift coefficient and angular speed of the relevant propeller, respectively.

Further, the input torque of attitude control for a quadrotor UAV can be obtained as

$$
\Gamma=\left[\begin{array}{c}
\Gamma_{\phi} \\
\Gamma_{\theta} \\
\Gamma_{\psi}
\end{array}\right]=\left[\begin{array}{cccc}
0 & b_{1} l & 0 & -b_{1} l \\
-b_{1} l & 0 & b_{1} l & 0 \\
-c_{1} & c_{1} & -c_{1} & c_{1}
\end{array}\right]\left[\begin{array}{c}
\omega_{1}^{2} \\
\omega_{2}^{2} \\
\omega_{3}^{2} \\
\omega_{4}^{2}
\end{array}\right],
$$

where $c_{1}$ is an aerodynamic constant, and $l$ is the distance from the motors to the center of gravity.

The control problem considered in this work is to design a control system to guarantee the trajectory tracking of a moving target for the quadrotor system with bounded uncertainties and disturbances.

\section{Controller Design}

It is assumed that we can gain the trajectory of the moving target by the vision sensor and calculated the velocity and acceleration of the target. The overall control objective for the quadrotor with a desired yaw angle $\psi_{d}$ is to track the desired trajectory of the target $\mathbf{P}_{d}=\left[x_{d}, y_{d}, z_{d}\right]^{\mathrm{T}}$. The speed and acceleration of the target can be defined as $\mathbf{V}_{d}$ and $\mathbf{a}_{d}$, respectively. The distance from the quadrotor to the target can be described by $\rho=\mathbf{P}-\mathbf{P}_{d}$. For a given expected tracking distance $\rho_{d}$, the error of the relative distance is given by $\mathbf{e}_{P}=\rho-\rho_{d}$. The purpose of target tracking control is to design a closed-loop controller to make the tracking error approach 0 .

In this section, the control system is composed of the position controller and attitude controller. Firstly, a position controller based on the artificial potential field and passive control is designed to obtain the desired position tracking for $\mathbf{P}_{d}$ and generate the desired roll and pitch references $\phi_{d}$ 
and $\theta_{d}$. Besides, an attitude controller based on the supertwisting sliding mode method with an improved reaching law is applied to make the aircraft track the desired attitude vector $\boldsymbol{\Theta}_{d}=\left[\phi_{d}, \theta_{d}, \psi_{d}\right]^{\mathrm{T}}$. The control structure is depicted in Figure 3.

3.1. Position Controller Design. According to (1), the translational error is obtained by

$$
\ddot{e}_{P}=\frac{\mathbf{U}_{P}+\mathbf{d}_{1}}{m}-g \mathbf{e}_{3}-\mathbf{a}_{d},
$$

where $\mathbf{U}_{P}=U_{1} \mathbf{R e}_{3}$ is the virtual control input.

We use the artificial potential field method to solve the position tracking. Firstly, the repulsion potential $J^{r}$ is constructed as a generalized Morse function [28]:

$$
J^{r}= \begin{cases}a \frac{b}{e^{(\|\boldsymbol{\rho}\| / \mathrm{c})}-e^{\left(\|\boldsymbol{\rho}\|_{\min } / c\right)},} & \|\boldsymbol{\rho}\| \in D, \\ 0, & \|\boldsymbol{\rho}\| \notin D,\end{cases}
$$

where $b$ and $c$ are constant, which determine the magnitude and velocity of the repulsion force, respectively. The gain coefficient of the potential field $a$ is a constant. $D=\left[\|\rho\|_{\min },\|\rho\|_{\max }\right.$ determines the action area of the artificial potential field. $\|\rho\|_{\min }>0$ represents the minimum safe distance, and $\|\rho\|_{\max }$ is expressed as the maximum distance.

In order to ensure that the artificial potential field has the correct equilibrium state $\left\|\rho_{d}\right\|$, the gravitational potential $J^{a}$ is defined as

$$
J^{a}= \begin{cases}\frac{1}{2} a \cdot k\|\boldsymbol{\rho}\|^{2}, & \|\boldsymbol{\rho}\| \in D, \\ 0, & \|\boldsymbol{\rho}\| \notin D,\end{cases}
$$

where $k$ indicates a positive constant that can adjust the strength of gravitational potential.

If the quadrotor can keep a certain distance to track the target, the velocity field of the artificial potential field at the equilibrium point is 0 . The values of parameters $k, b$, and $c$ will be satisfied as

$$
-k\left(\left\|\boldsymbol{\rho}_{d}\right\|\right)+\frac{b}{c} \frac{1}{\left(e^{\left(\left\|\rho_{d}\right\| / c\right)}-e^{\left(\| \boldsymbol{\rho}_{\min } / c\right)}\right)^{2}} e^{\left\|\boldsymbol{\rho}_{d}\right\| / c}=0 .
$$

When $\left\|\rho_{d}\right\| \leq\|\rho\| \leq\|\rho\|_{\max }$, the gravitational force has a great effect on the convergence in long distances. When $\|\rho\|_{\text {min }} \leq\|\rho\| \leq\left\|\rho_{d}\right\|$, the repulsive force plays a leading role in short distances. When $\|\rho\|>\|\rho\|_{\max }$, the potential field no longer works. When $\|\rho\|=\left\|\rho_{d}\right\|$, the two forces are just equal and the potential field reaches equilibrium.

By combining repulsive potential and gravitational potential, the velocity field function between UAV and target for $\|\rho\| \in D$ can be determined as

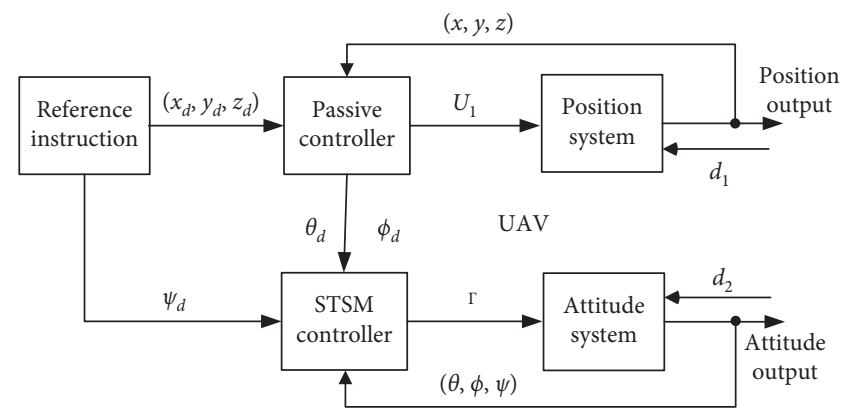

FIGURE 3: Structure of the quadrotor control system.

$$
\begin{array}{r}
\mathbf{v}_{f}=-\nabla J^{r}-\nabla J^{a} \\
=a\left[-k\|\boldsymbol{\rho}\|+\frac{b}{c} \frac{1}{\left(e^{(\|\boldsymbol{\rho}\| / c)}-e^{\left(\|\boldsymbol{\rho}\|_{\min } / c\right)}\right)^{2}} e^{\|\boldsymbol{\rho}\| / c}\right] \frac{\boldsymbol{\rho}}{\|\boldsymbol{\rho}\|} .
\end{array}
$$

Definition 1. Considering nonlinear systems,

$$
\left\{\begin{array}{l}
\dot{x}=f(x, u), \\
y=h(x) .
\end{array}\right.
$$

For all $x \in R^{n}$ and $u \in R^{m}$, fis a locally Lipschitz function and $f(0,0)=0$, and $h(x)$ is continuous and $h(0)=0$. If a differentiable positive definite function $V(x)$ satisfies

$$
u^{T} y \geq \dot{V}=\frac{\partial V}{\partial x} f(x, u)
$$
[35].

then, the abovementioned nonlinear system is passive

The velocity error can be set as

$$
\dot{e}_{P}=\dot{P}-\mathbf{v}_{d}-\dot{\rho}_{d}=\mathbf{v}_{e} .
$$

The velocity field is used to calculate the relative velocity between UAV and target. The relative velocity is defined as

$$
\mathbf{v}_{r}=\mathbf{v}_{e}-\mathbf{v}_{f}+\alpha \mathbf{e}_{P}, \quad \alpha>0 .
$$

Based on (15), the velocity error can be derived as

$$
\dot{e}_{P}=-\alpha \mathbf{e}_{P}+\mathbf{v}_{r}+\mathbf{v}_{f} .
$$

The derivative of $v_{r}$ can be derived as

$$
\dot{v}_{r}=\dot{v}_{e}-\dot{v}_{f}+\alpha \dot{e}_{p}=\frac{\mathbf{U}_{p}}{m}-g \mathbf{e}_{3}+\frac{\mathbf{d}_{1}}{\mathrm{~m}}-\mathbf{a}_{t}-\dot{v}_{f}+\alpha \dot{e}_{p} .
$$

To ensure accurate tracking of moving targets, a virtual control input of the position controller is designed as

$$
\mathbf{U}_{p}=m g \mathbf{e}_{3}-\widehat{d}_{1}+m\left(\ddot{P}_{d}-\lambda_{1} \mathbf{v}_{r}+\dot{v}_{f}+\mathbf{u}-\alpha \dot{e}_{p}\right),
$$

where $\lambda_{1}>0, \hat{d}_{1}$ is the estimate of $d_{1}$, and $u$ is the virtual input of the passive system.

Substituting (18) into (17) yields 


$$
\begin{aligned}
\dot{v}_{r} & =-g \mathbf{e}_{3}-\widehat{d}_{1}-\lambda_{1} \mathbf{v}_{r}+\dot{v}_{f}-\alpha \dot{e}_{p}+g \mathbf{e}_{3}-\dot{v}_{f}+\mathbf{d}_{1}+\alpha \dot{e}_{p}+\mathbf{u} \\
& =-\lambda_{1} \mathbf{v}_{r}+\tilde{d}_{1}+\mathbf{u},
\end{aligned}
$$

where the error $\widetilde{d}_{1}=\mathbf{d}_{1}-\widehat{d}_{1}$.

For the tracking system, the structure of the cascaded passive system is shown in Figure 4. The total input of the system is $\widetilde{d}_{1}+\mathbf{u}$, and the output is $\mathbf{e}_{p}$. According to passive theory, the passivity of the system can improve the accuracy of the position tracking and avoid the local minimum of the artificial potential field near the equilibrium point. Thus, the stability of the system will be improved.

Theorem 1. Consider the quadrotor translational dynamics described in (8). The whole position tracking system acts as a cascade of the drive system (17) and driven system (20). If the relative velocity and the virtual control input are designed according to (16) and (19), the control strategy can guarantee that the position control system is passive and globally stable at the origin.

Proof. If a candidate storage function is set as

$$
\mathbf{V}_{3}\left(\mathbf{e}_{p}, \mathbf{v}_{r}\right)=\mathbf{V}_{1}\left(\mathbf{e}_{p}\right)+\mathbf{V}_{2}\left(\mathbf{v}_{r}\right),
$$

where $\mathbf{V}_{1}=(1 / 2) \mathbf{e}_{P}^{T} \mathbf{e}_{P}, \mathbf{V}_{2}=(1 / 2) \mathbf{v}_{r}^{T} \mathbf{v}_{r}$.

According to (11) and (12), we can get

$\mathbf{e}_{P} \mathbf{v}_{f}=a \frac{\boldsymbol{\rho}\left(\boldsymbol{\rho}-\boldsymbol{\rho}_{d}\right)}{\|\boldsymbol{\rho}\|}\left[-k\|\boldsymbol{\rho}\|+\frac{b}{c} \frac{1}{\left(e^{(\|\boldsymbol{\rho}\| / c)}-e^{\left(\|\boldsymbol{\rho}\|_{\min } / c\right)}\right)^{2}} e^{\|\boldsymbol{\rho}\| / c}\right]=T \cdot Q$,

where $T=a\left(\rho\left(\rho-\rho_{d}\right) /\|\rho\|\right), Q=\left[-k\|\rho\|+b / c\left(1 /\left(e^{(\|\rho\| / c)}-\right.\right.\right.$ $\left.\left.\left.e^{\left(\|\rho\|_{\min } / c\right)}\right)^{2}\right) e^{(\|\rho\| / c)}\right]$.

If $\|\rho\| \geq\left\|\rho_{d}\right\|$, then $T \geq 0, Q \leq 0$. If $\|\rho\|<\left\|\rho_{d}\right\|$, then $T<0$, $Q>0$. It can be derived as $\mathbf{e}_{P}^{T} \mathbf{v}_{f} \leq 0$.

The derivative of $\mathbf{V}_{1}$ can be given by

$$
\dot{V}_{1}=\mathbf{e}_{P}^{T} \cdot \dot{e}_{P}=\mathbf{e}_{P}^{T} \mathbf{v}_{r}-\alpha \mathbf{e}_{P}^{T} \mathbf{e}_{P}+\mathbf{e}_{P}^{T} \mathbf{v}_{f} \leq \mathbf{e}_{P}^{T} \mathbf{v}_{r} .
$$

Then, the system is passive to $\mathbf{e}_{P}$ and $\mathbf{v}_{r}$.

The derivative of $\mathbf{V}_{2}$ can be written as

$$
\dot{V}_{2}=\mathbf{v}_{r}^{T} \cdot \dot{v}_{r}=-\lambda_{1} \mathbf{v}_{r}^{T} \mathbf{v}_{r}+\mathbf{v}_{r}^{T}\left(\tilde{d}_{1}+\mathbf{u}\right) \leq \mathbf{v}_{r}^{T}\left(\tilde{d}_{1}+\mathbf{u}\right) \text {. }
$$

Thus, the system is passive to $\mathbf{v}_{r}$ and $\widetilde{d}_{1}+\mathbf{u}$.

The derivative of $\mathbf{V}_{3}$ can be solved as

$$
\begin{aligned}
\dot{V}_{3} & =\mathbf{e}_{p} \mathbf{v}_{r}^{T}+\mathbf{v}_{r}^{T}\left(\widetilde{d}_{1}+\mathbf{u}\right)+\mathbf{e}_{P}^{T} \mathbf{v}_{f}-\alpha \mathbf{e}_{p}^{T} \mathbf{e}_{p}-\lambda_{1} \mathbf{v}_{r}^{T} \mathbf{v}_{r} \\
& \leq \mathbf{e}_{p} \mathbf{v}_{r}^{T}+\mathbf{v}_{r}^{T}\left(\widetilde{d}_{1}+\mathbf{u}\right)-\alpha \mathbf{e}_{p}^{T} \mathbf{e}_{p}-\lambda_{1} \mathbf{v}_{r}^{T} \mathbf{v}_{r} \\
& \leq \mathbf{e}_{p} \mathbf{v}_{r}^{T}+\mathbf{v}_{r}^{T}\left(\widetilde{d}_{1}+\mathbf{u}\right)=\mathbf{v}_{r}^{T}\left(\mathbf{e}_{p}+\widetilde{d}_{1}+\mathbf{u}\right) .
\end{aligned}
$$

The feedback control is designed as

$$
\mathbf{u}=-\mathbf{e}_{p}-\beta \mathbf{v}_{r},
$$

where $\beta>0$; then,

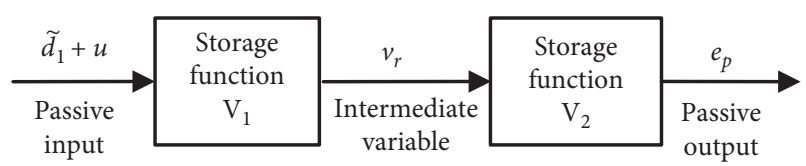

Figure 4: Cascade passive structure of quadrotor.

$$
\dot{V}_{3}=\mathbf{v}_{r}^{T}\left(\widetilde{d}_{1}-\beta \mathbf{v}_{r}\right)=\mathbf{v}_{r}^{T} \widetilde{d}_{1}-\beta \mathbf{v}_{r}^{T} \mathbf{v}_{r} \leq \mathbf{v}_{r}^{T} \widetilde{d}_{1} .
$$

Taking into account uncertainties and external disturbances, the storage function $\mathbf{V}$ is chosen as

$$
\mathbf{V}=\mathbf{V}_{3}+\frac{1}{2} \mathbf{d}_{1}^{T} \widetilde{d}_{1} .
$$

The adaptive law is constructed as

$$
\dot{\hat{d}}_{1}=-\mathbf{v}_{r} \text {. }
$$

Because $\tilde{d}_{1}$ is a slow time-varying signal, the derivative of $\mathrm{V}$ can be solved as

$$
\begin{aligned}
\dot{V} & =\mathbf{v}_{r} \tilde{d}_{1}^{T}-\beta \mathbf{v}_{r}^{T} \mathbf{v}_{r}+\tilde{d}_{1}^{T} \dot{\tilde{d}}_{1}=\mathbf{v}_{r} \tilde{d}_{1}^{T}-\beta \mathbf{v}_{r}^{T} \mathbf{v}_{r}-\tilde{d}_{1}^{T} \dot{\hat{d}}_{1} \\
& =-\beta \mathbf{v}_{r}^{T} \mathbf{v}_{r} \leq 0 .
\end{aligned}
$$

Therefore, it is shown that the controller can force the states of the system with bounded disturbances and uncertainties to converge asymptotically to origin.

We define the virtual control input $U_{P}=\left[U_{x}, U_{y}, U_{z}\right]^{\mathrm{T}}$, satisfying

$$
\left[\begin{array}{l}
U_{x} \\
U_{y} \\
U_{z}
\end{array}\right]=U_{1}\left[\begin{array}{ccc}
C_{\theta} C_{\psi} & S_{\theta} S_{\phi} C_{\psi}-C_{\phi} S_{\psi} & C_{\phi} S_{\theta} C_{\psi}+S_{\phi} S_{\psi} \\
C_{\theta} S_{\psi} & S_{\phi} S_{\theta} S_{\psi}+C_{\phi} C_{\psi} & C_{\phi} S_{\theta} S_{\psi}-S_{\phi} C_{\psi} \\
-S_{\theta} & S_{\phi} C_{\theta} & C_{\phi} C_{\theta}
\end{array}\right]\left[\begin{array}{l}
0 \\
0 \\
1
\end{array}\right] .
$$

The above matrix expression can be obtained by

$$
\left\{\begin{array}{l}
U_{x}=U_{1}\left(C_{\phi} S_{\theta} C_{\psi}+S_{\phi} S_{\psi}\right) \\
U_{y}=U_{1}\left(C_{\phi} S_{\theta} S_{\psi}-S_{\phi} C_{\psi}\right) \\
U_{z}=U_{1} C_{\phi} C_{\theta} .
\end{array}\right.
$$

According to (32), the actual control input $U_{1}$ can be solved as

$$
U_{1}=\frac{U_{z}}{C_{\phi_{d}} C_{\theta_{d}}} .
$$

Substituting $U_{1}$ into $U_{x}$ and $U_{y}$, we can get two desired attitude angles from (32); that is,

$$
\left\{\begin{array}{l}
\theta_{d}=\arctan \left(\frac{U_{x} C_{\psi_{d}}+U_{y} S_{\psi_{d}}}{U_{z}}\right), \\
\phi_{d}=\arctan \left(C_{\theta_{d}} \frac{U_{x} S_{\psi_{d}}-U_{y} C_{\psi_{d}}}{U_{z}}\right) .
\end{array}\right.
$$


3.2. Attitude Controller Design. In this section, the attitude controller is designed by using the super-twisting sliding mode control with an improved reaching law. The objective of the controller is to ensure the convergence of the attitude angles to the desired trajectories.

Considering both model uncertainties and external disturbances in the attitude subsystem, (2) can be written as

$$
\mathbf{J}_{0} \ddot{\Theta}=-\mathbf{C}_{0} \dot{\Theta}_{d}+\Gamma+\mathbf{d}_{2}-\mathbf{J}_{\Delta} \ddot{\Theta}-\mathbf{C}_{\Delta} \dot{\Theta},
$$

where $\mathbf{J}=\mathbf{J}_{0}+\mathbf{J}_{\Delta}, \mathbf{C}=\mathbf{C}_{0}+\mathbf{C}_{\Delta}, \mathbf{C}_{0}=0.9 \mathbf{C}$.

$$
\mathbf{J}_{0}=\left[\begin{array}{ccc}
I_{x x} & 0 & 0 \\
0 & I_{x x}+I_{y y} & 0 \\
0 & 0 & I_{x x}+I_{y y}+I_{z z}
\end{array}\right] \text {. }
$$

The vector $d$ denote the norm-bounded model uncertainty and external disturbances in rotational motions as

$$
\mathbf{d}=\mathbf{d}_{2}-\mathbf{J}_{\Delta} \ddot{\Theta}-\mathbf{C}_{\Delta} \dot{\Theta},
$$

where $\|\mathbf{d}\| \leq D_{1}$.

The dynamic model of attitude system can be transformed into

$$
\mathbf{J}_{0} \ddot{\Theta}+\mathbf{C}_{0} \dot{\Theta}=\Gamma+\mathbf{d} .
$$

The state-space form of this model can be given by

$$
\left\{\begin{array}{l}
\dot{\Theta}_{1}=\boldsymbol{\Theta}_{2}, \\
\dot{\Theta}_{2}=f(\boldsymbol{\Theta})+g(\boldsymbol{\Theta}) \Gamma+\mathbf{d},
\end{array}\right.
$$

where the vector $f(\Theta)=-\mathbf{J}_{0}^{-1} \mathbf{C} \dot{\Theta}$ and $g(\Theta)=\mathbf{J}_{0}^{-1}$.

The tracking error of attitude $e_{1}$ is defined as

$$
\mathbf{e}_{1}=\Theta_{1 d}-\Theta_{1} .
$$

The angular velocity tracking error is given by

$$
\mathbf{e}_{2}=\dot{e}_{1} \text {. }
$$

The sliding mode manifold is designed as

$$
s=e_{2}+\lambda e_{1}
$$

where $\mathbf{s}=\left[\begin{array}{lll}s_{\phi} & s_{\theta} & s_{\psi}\end{array}\right]^{\mathrm{T}}, \lambda>0$.

The reaching law of the sliding mode manifold is chosen as

$$
\begin{aligned}
\dot{s}= & -k_{1} \int_{0}^{s} \operatorname{sgn}(\mathbf{s}(\tau)) \mathrm{d} \tau-k_{2}|\mathbf{s}|^{(1 / 2)} \operatorname{sgn}(\mathbf{s}) \\
& -\left(2 k_{4}+k_{1}\right) \mathbf{s}-k_{3} \operatorname{sgn}(\mathbf{s}),
\end{aligned}
$$

where the gain coefficient $k_{1}, k_{2}$, and $k_{4}$ are positive constant, $k_{3}>D_{1} \geq\|\mathbf{d}\|$.

The improved reaching law adds the proportion term and the velocity term. The law provides a power convergence rate in the reaching phase and reduces the approaching time to the sliding surface. In addition, the combined terms can guarantee that the system state reaches the sliding mode surface with a good dynamic performance.

Substituting (42) and (43) into (39) leads to

$$
\begin{aligned}
\Gamma= & g^{-1}(\boldsymbol{\Theta})\left\{-f(\boldsymbol{\Theta})+\ddot{\Theta}_{1 d}+\lambda \dot{e}_{1}+\left(2 k_{4}+k_{1}\right) \mathbf{s}+\mathbf{d}\right. \\
& \left.+k_{1} \int_{0}^{s} \operatorname{sgn}(\mathbf{s}) \mathrm{d} \tau+k_{2}|\mathbf{s}|^{(1 / 2)} \operatorname{sgn}(\mathbf{s})+k_{3} \operatorname{sgn}(\mathbf{s})\right\} .
\end{aligned}
$$

Theorem 2. Consider the quadrotor rotational dynamics described in (2). If the sliding mode manifold and control input are designed according to (42) and (43), the control strategy can ensure that the attitude tracking error $e_{1}$ converges to zero.

Proof. If a candidate Lyapunov function is selected as

$$
W=\frac{1}{2} \mathbf{e}_{1}^{T} \mathbf{e}_{1}+\frac{1}{2} \mathbf{s}^{T} \mathbf{s}
$$

the derivative of $W$ is computed as

$$
\begin{aligned}
\dot{W} & =\mathbf{e}_{1}^{T} \mathbf{e}_{2}+\mathbf{s}^{T}\left[-k_{1} \int_{0}^{t} \operatorname{sgn}(\mathbf{s}) \mathrm{d} \tau-\left(2 k_{4}+k_{1}\right) \mathbf{s}-k_{2}|\mathbf{s}|^{(1 / 2)} \operatorname{sgn}(\mathbf{s})-k_{3} \operatorname{sgn}(\mathbf{s})+\mathbf{d}\right] \\
& =\mathbf{e}_{1}^{T} \mathbf{e}_{2}-2 k_{4} \mathbf{s}^{T} \mathbf{s}-k_{2}|\mathbf{s}|^{(3 / 2)}-k_{3}|\mathbf{s}|+\mathbf{s}^{T} \mathbf{d} \leq \mathbf{e}_{1}^{T} \mathbf{e}_{2}-2 k_{4} \mathbf{s}^{T} \mathbf{s}-k_{2}|\mathbf{s}|^{(3 / 2)} \\
& \leq-\left[k_{4} \lambda^{2} \mathbf{e}_{1}^{T} \mathbf{e}_{1}+\left(2 k_{4} \lambda-1\right) \mathbf{e}_{1}^{T} \mathbf{e}_{2}+k_{4} \mathbf{e}_{2}^{T} \mathbf{e}_{2}\right]-k_{4} \mathbf{s}^{T} \mathbf{s}-k_{2}|\mathbf{s}|^{(3 / 2)} \leq-\left[\begin{array}{ll}
\mathbf{e}_{1} & \mathbf{e}_{2}
\end{array}\right] Q\left[\begin{array}{ll}
\mathbf{e}_{1} & \mathbf{e}_{2}
\end{array}\right]^{T}-k_{4} \mathbf{s}^{T} \mathbf{s}-k_{2}|\mathbf{s}|^{(3 / 2)}
\end{aligned}
$$

where $Q=\left[\begin{array}{cc}k_{4} \lambda^{2} & k_{4} \lambda-1 / 2 \\ k_{4} \lambda-1 / 2 & k_{4}\end{array}\right]$.

The matrix $Q$ can be calculated as $|\mathbf{Q}|=k_{4} \lambda-(1 / 4)$. If appropriate values of $k_{2}, k_{4}$, and $\lambda$ are selected to make $|\mathbf{Q}|>0$ and $\dot{W} \leq 0$, the sliding surface will converge to 0 . It can be concluded that the proposed attitude controller can guarantee that the attitude angle asymptotically converges to the desired values. 


\section{Simulation and Analysis}

Trajectory tracking simulations of the quadrotor have been carried out to verify the validity and efficiency of the proposed control method. Besides, a synthesis controller based on second-order SMC (2-SMC) [36] has been chosen for the comparison.

The parameter values of the quadrotor model are as follows: $m=0.5 \mathrm{~kg}, b_{1}=5 \mathrm{Ns}^{2}, c_{1}=2 \mathrm{Nms}^{2}, l=0.5 \mathrm{~m}, g=9.8 \mathrm{~m} / \mathrm{s}^{2}$, $I_{x x}=I_{y y}=0.004 \mathrm{kgm}^{2}, I_{z z}=0.008 \mathrm{kgm}^{2}$, and the yaw angle $\psi_{d}=\pi / 3$. The external disturbances are considered as $d_{1}=\left[\begin{array}{lll}0.1 \sin (0.1 \pi t), & 0.1 \cos (0.1 \pi t), \quad 0.1 \cos (0.1 \pi t)\end{array}\right]^{T}$ and $d_{2}=$ $[0.3 \sin (0.1 \pi t)+0.1,0.4 \cos (0.1 \pi t)+0.1,0.5 \sin (0.1 \pi t)+0.2]^{T}$.

In order to further evaluate the robustness of the proposed control method, another simulation has been executed for the system under external disturbances and parametric uncertainties. The parameter uncertainties are considered as $\pm 30 \%$ in the mass $m$ and inertia matrix $I_{x x}, I_{y y}$, and $I_{z z}$ respectively.

The controller parameters of 2-SMC are given as follows: $c_{z}=1, c_{\psi}=1, \varepsilon_{1}=1, \varepsilon_{2}=1, \varepsilon_{3}=0.5, \varepsilon_{4}=0.5, \eta_{1}=1, \eta_{2}=1$, $\eta_{3}=4, \eta_{4}=4, c_{1}=10 m_{\mathrm{s}} /\left(u_{1} \cos \phi \cos \psi\right), c_{2}=5 m_{\mathrm{s}} /\left(u_{1} \cos \phi \cos \psi\right)$, $c_{3}=1, c_{4}=5, c_{5}=-10 m_{\mathrm{s}} /\left(u_{1} \cos \psi\right), c_{6}=-5 m_{\mathrm{s}} /\left(u_{1} \cos \psi\right), c_{7}=1$, and $c_{8}=5$.

The parameters of the control method based on PC and STSM (PC\&STSM) are adjusted as $\|\rho\|_{\min }=1,\left\|\rho_{d}\right\|=2$, $\|\rho\|_{\max }=5, k=10, a=1, b=16.83, c=2, \alpha=0.8, \lambda_{1}=4, \beta=2$, $k_{1}=0.5, k_{2}=1, k_{3}=3, k_{4}=2$, and $\lambda=10$. According to the tracking target, selecting methods of controller parameters will be given as follows.

(1) First, it is assumed that the expected distance between the aircraft and the moving target $\left\|\rho_{d}\right\|=2$. The gain coefficient $a$ will be chosen as $a=1$ to balance the potential field force. When $2 \leq\|\rho\| \leq 5$, we choose $k=10$ to get a large gravitational force. When $1 \leq\|\rho\| \leq 2$, we choose $c=2$ to get a slightly large repulsive force. According to (11), we can get $b=16.83$ at the equilibrium point of the artificial potential field

(2) The parameters $\alpha$ and $\beta$ are in connection with the convergence rate of the relative velocity and position error. The parameter $\lambda_{1}$ is related to the external disturbances in translational motion. Through several simulation attempts, we choose $\alpha=0.8, \beta=2$, and $\lambda_{1}=4$ in order to improve the convergence rate and accuracy

(3) When $s=0, \dot{e}_{1}=-\lambda e_{1}$. If any attitude angle error needs to converge to 0.01 in $0.5 \mathrm{~s}, \lambda$ should be chosen as $\lambda=10$. The parameters $k_{1}, k_{2}$, and $k_{4}$ are in connection with the accuracy and convergence speed of attitude angle error. According to (43) and (47), $k_{1}>0, k_{2}>0$, and $k_{4} \lambda>0.25$. Through several simulation attempts, we choose $k_{1}=0.5, k_{2}=1$, and $k_{4}=2$ to obtain fast convergence and small overshoot. Finally, the parameter $k_{3}$ is related to the robustness of the system, and $k_{3}$ will be adjusted according to $k_{3}>\|\mathbf{d}\|$

In order to show the effectiveness of the proposed strategy, we select two tracking scenarios for simulation.
4.1. Ground Moving Target. The initial position and attitude angle values of the quadrotor are $[0,0,1.5] m$ and $[0.2,0.2$, $0.5] \mathrm{rad}$. The trajectory of a moving ground target can be described as

$$
\mathbf{P}_{d}=\left[4 \mathrm{t} 4 \sin \left(\frac{t}{2}\right) 2\right] .
$$

Simulation results of trajectory tracking are depicted in Figure 5. Figure 5(a) presents tracking results from the two control methods in $3 \mathrm{D}$ space. It can be observed that the PC\&STSM method can make the quadrotor track the target accurately at a fixed distance, whereas the 2-SMC approach makes it slightly off the desired track at the beginning. As shown in Figure 5(b), the position tracking from APF\&PC achieves null steady-state error for all positions in $1.8 \mathrm{~s}$, while the 2 -SMC controller takes $3.8 \mathrm{~s}$ to get the steady state. Figure 5(c) shows that the STSM controller has a fast convergence rate than that from 2-SMC. Compared with 2SMC, the improved reaching law greatly improves the convergence performance. The comparisons of convergence time and overshoot between the two methods are listed in Tables 1 and 2.

As shown in Figure 6, the simulation results of trajectory tracking demonstrate the control quality of the proposed controller in terms of disturbances and uncertainties.

It can be seen from Figure 6(a) that the PC\&STSM controller can still track accurately the trajectory of the moving ground target when the mass and inertia matrixes of the quadrotor change by $\pm 30 \%$. According to Figure 6(b), the position tracking generated by the controller converges to the set position in $2 \mathrm{~s}$ even if the disturbances and uncertainties are considered. Besides, the figure also shows that parameter uncertainties have few effects on position control. From Figure 6(c), it can be observed that attitude angles converge in a short time and keep the desired values all the time although uncertainties and disturbances cause overshoot. The simulation results have illustrated that the PC\&STSM controller has good robustness and tracking performance in the case of external disturbances and parameter uncertainties.

4.2. Aerial Moving Target. The initial position and attitude angle values of the quadrotor are $[0,0,-1] m$ and $[0,0.2,0.5]$ $\mathrm{rad}$. The trajectory of a moving aerial target can be described as

$$
\mathbf{P}_{d}=\left[\frac{1}{2} \cos \left(\frac{t}{2}\right) \frac{1}{2} \sin \left(\frac{t}{2}\right) 2+\frac{t}{10}\right] .
$$

According to Figure 7(a), it can be concluded that the aerial target tracking control system can meet the tracking performance, and the PC\&STSM has a better tracking effect than 2-SMC. From Figure 7(b), it can be observed that APF\&PC is insensitive to disturbances and has a faster convergence speed in position tracking. In contrast, 2-SMC has a longer adjustment time. Figure 7(c) shows that STSM achieves zero steady-state error with a small overshoot between $0 \mathrm{rad}$ and $0.09 \mathrm{rad}$, while 2 -SMC has a bigger amplitude oscillation in attitude tracking. The response speed of STSM is faster than that of 2-SMC, which is helpful 


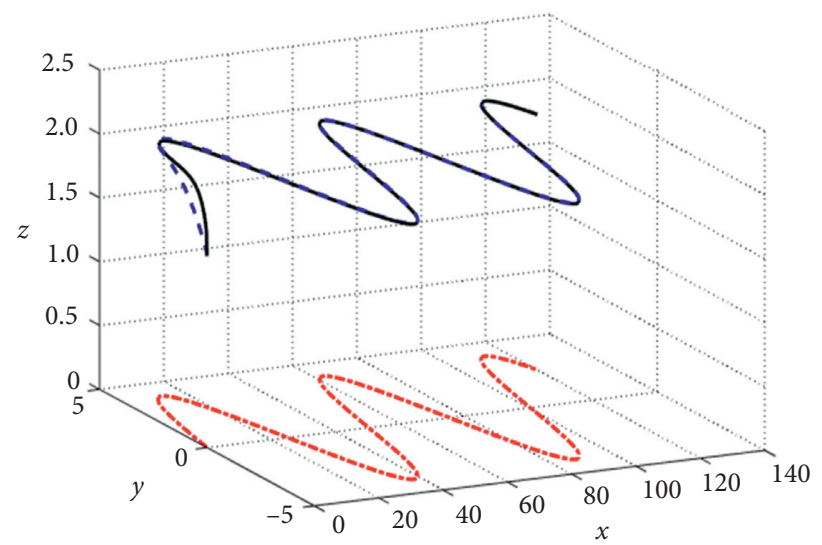

...- Target trajectory

- PC \& STSM tracking

- - - 2-SMC tracking

(a)

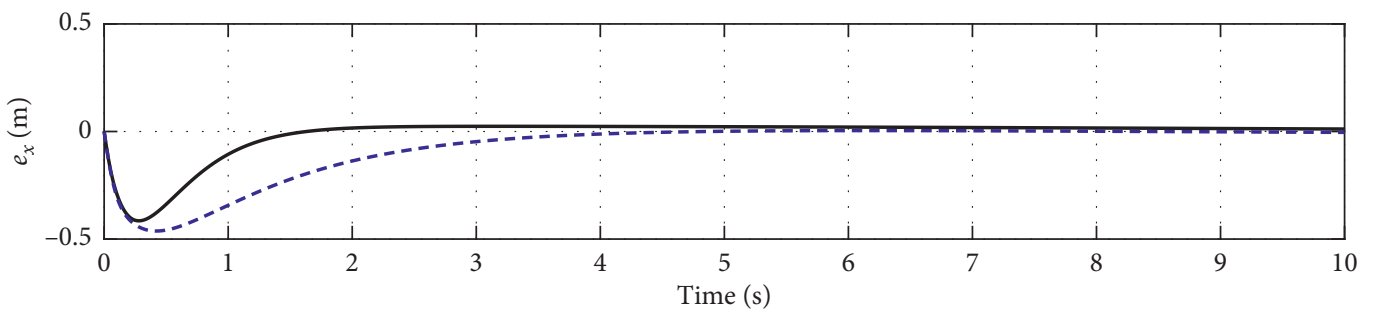

APF \& PC tracking

- - - 2-SMC tracking

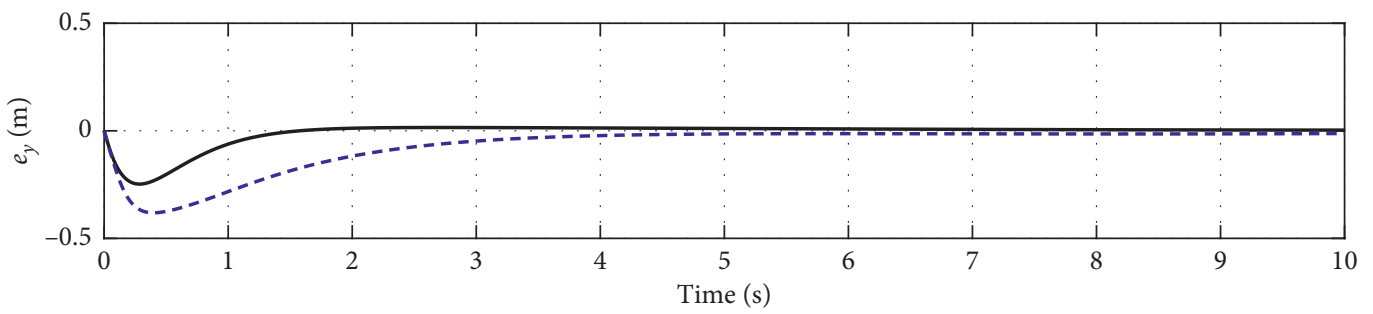

- APF \& PC tracking

-- 2-SMC tracking

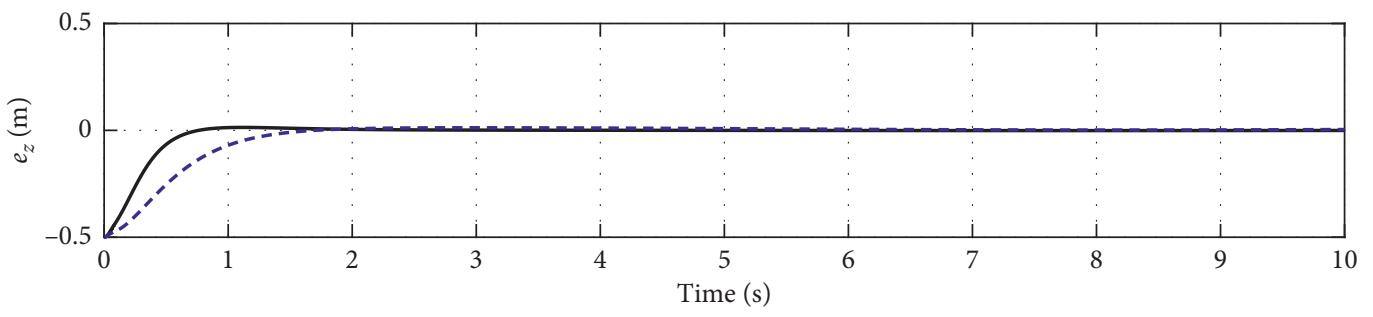

- APF \& PC tracking

-- - 2-SMC tracking

(b)

Figure 5: Continued. 


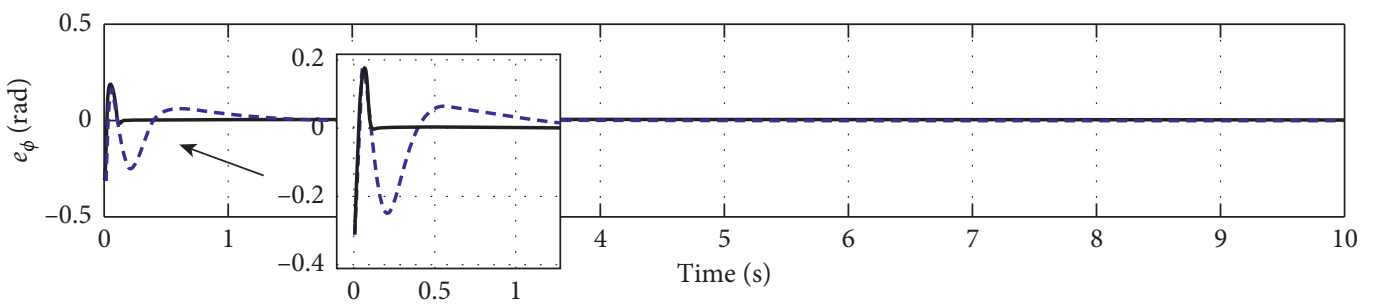

$\operatorname{STSM} \phi$

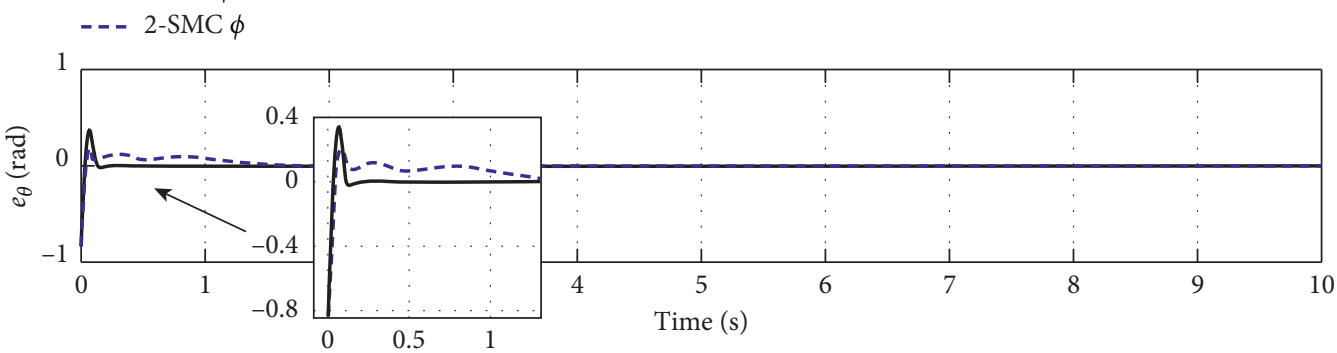

$\operatorname{STSM} \theta$

- - - 2-SMC $\theta$

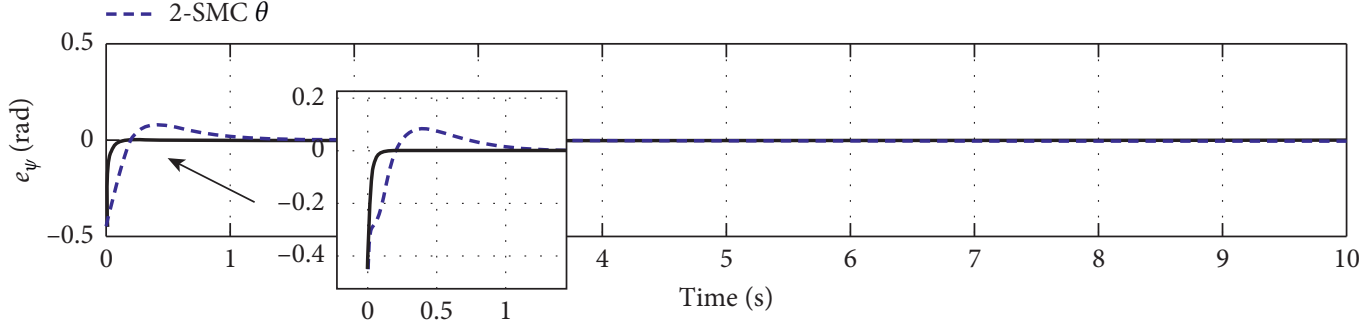

$-\operatorname{STSM} \psi$

$--\mathbf{- S M C} \psi$

(c)

FIGURE 5: Ground moving target tracking. (a) Trajectory tracking. (b) Position tracking error. (c) Attitude tracking error.

TABle 1: Convergence time of tracking error.

\begin{tabular}{lcccccc}
\hline Method & $\mathrm{x}(\mathrm{s})$ & $\mathrm{y}(\mathrm{s})$ & $\mathrm{z}(\mathrm{s})$ & $\phi(\mathrm{s})$ & $\boldsymbol{\psi}(\mathrm{s})$ & 0.4 \\
\hline PC\&STSM & 1.8 & 1.8 & 0.9 & 0.4 & 0.25 \\
2-SMC & 3.8 & 3.8 & 1.8 & 1.5 & 1.5 & 1.2 \\
\hline
\end{tabular}

TABLE 2: Overshoot of tracking error.

\begin{tabular}{lllllll}
\hline Method & $x$ & $y$ & $z$ & $\phi$ & $\theta$ & \\
\hline PC\&STSM & 0 & 0 & 0 & 0.18 & 0.38 & 0 \\
2-SMC & 0 & 0 & 0 & 0.18 & 0.24 & 0.1 \\
\hline
\end{tabular}

to flexibly move in the air. The comparisons of convergence time and overshoot between the two methods are listed in Tables 3 and 4.

As presented in Figures 6 and 8, the convergence of position and attitude state is slightly affected by the variation of system parameters, but the proposed controller can achieve zero steady-state error and compensate for external disturbances and parameter uncertainties. The simulation results verify the robustness of the proposed control scheme.

Numerical simulation indicated that the controller based on PC\&STSM can trace the moving target effectively and has a good dynamic performance. Compared with the 2-SMC method, the proposed controller has a better performance in tracking precision and robustness. 


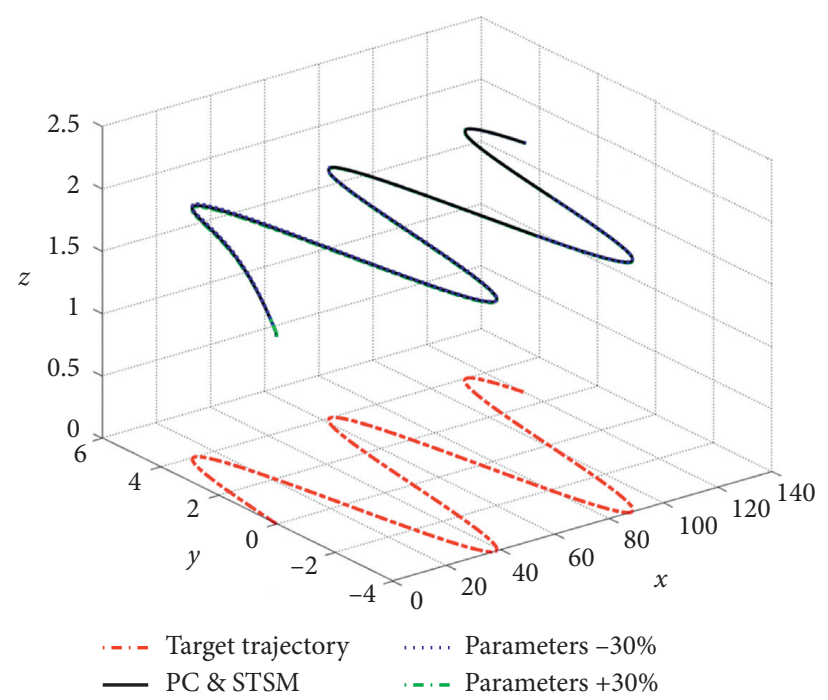

(a)

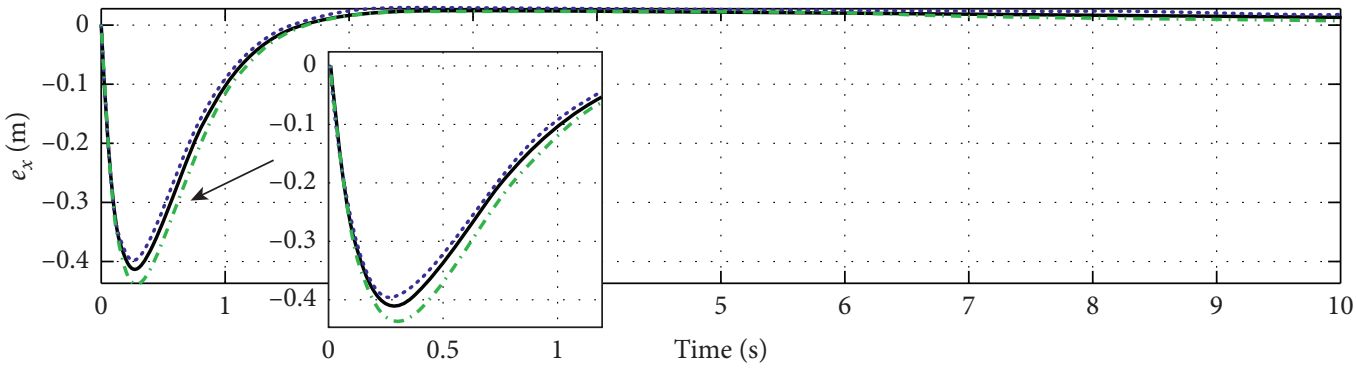

- APF \& PC

..... Parameters $-30 \%$

. - . - Parameters $+30 \%$

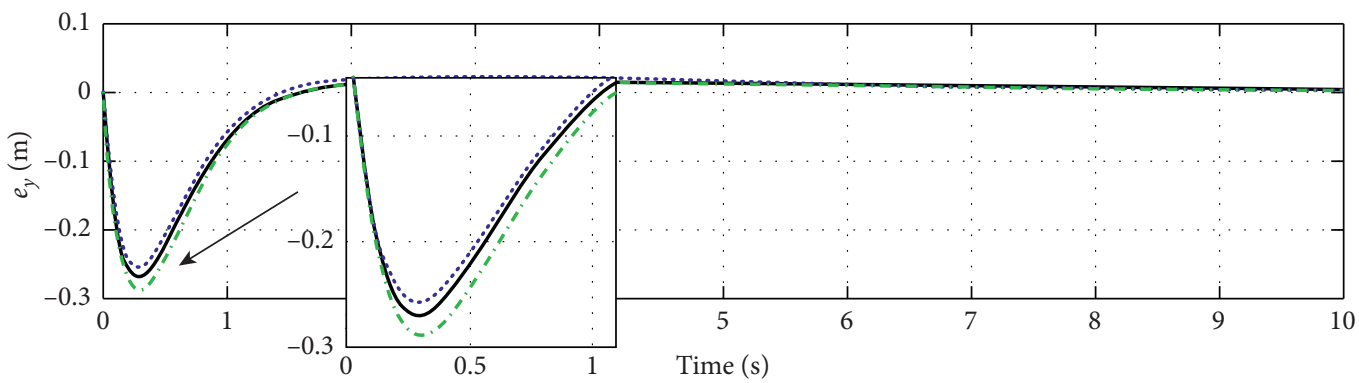

- APF \& PC

..... Parameters $-30 \%$

. . . - Parameters $+30 \%$

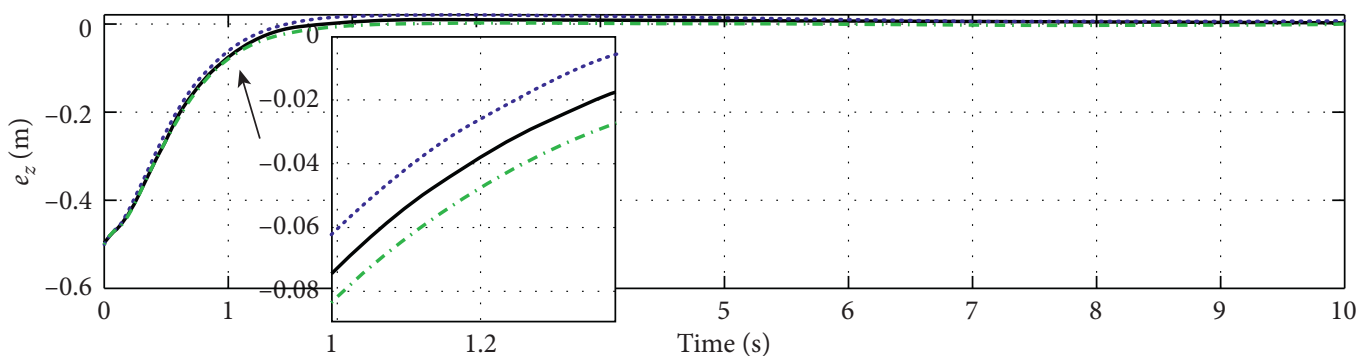

- APF \& PC

..... Parameters $-30 \%$

. . . - Parameters $+30 \%$

Figure 6: Continued. 


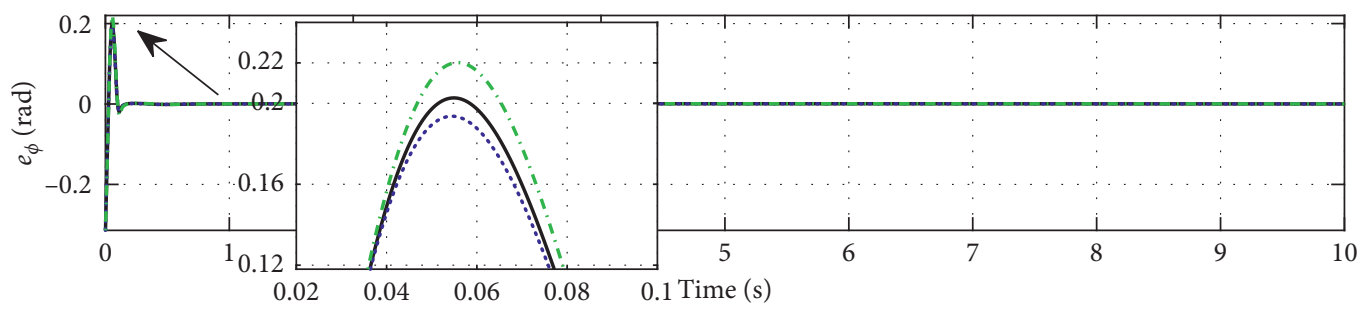

— STSM

..... Parameters $-30 \%$

. - . - Parameters $+30 \%$

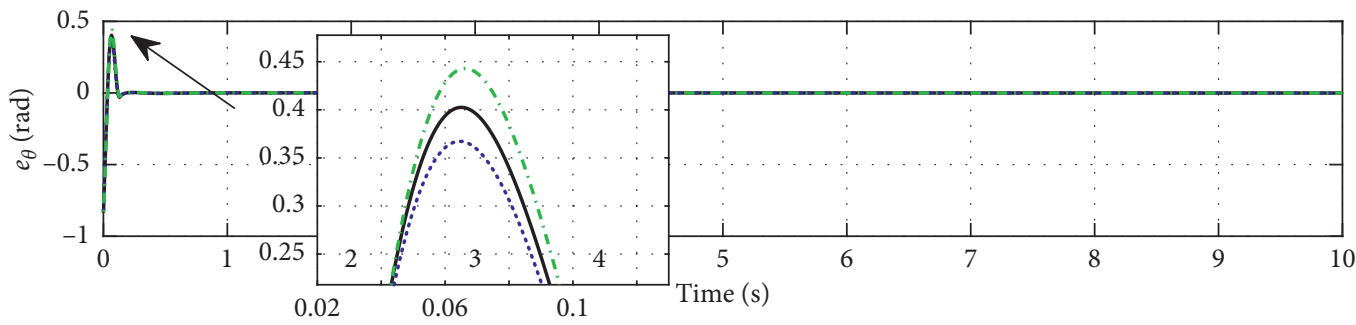

- STSM

..... Parameters $-30 \%$

...- Parameters $+30 \%$

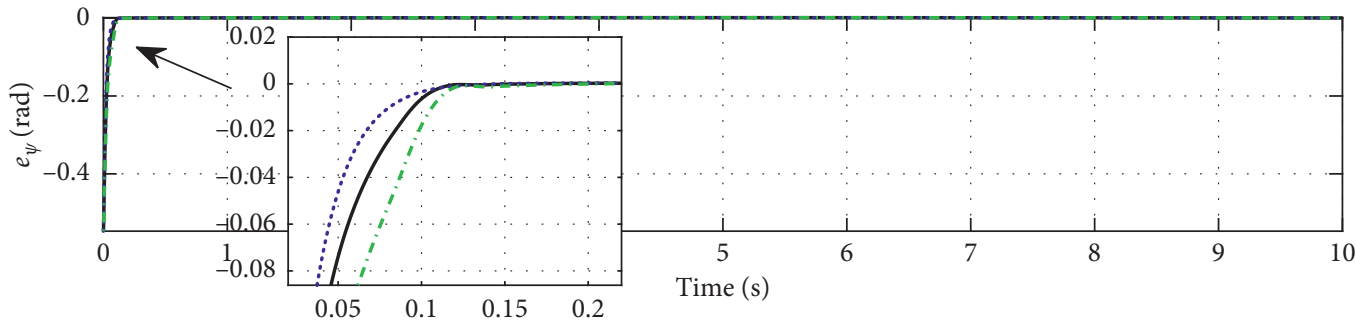

- STSM

..... Parameters $-30 \%$

. - . - Parameters $+30 \%$

(c)

Figure 6: Target tracking with uncertainties and disturbances. (a) Trajectory tracking. (b) Position tracking error. (c) Attitude tracking error.

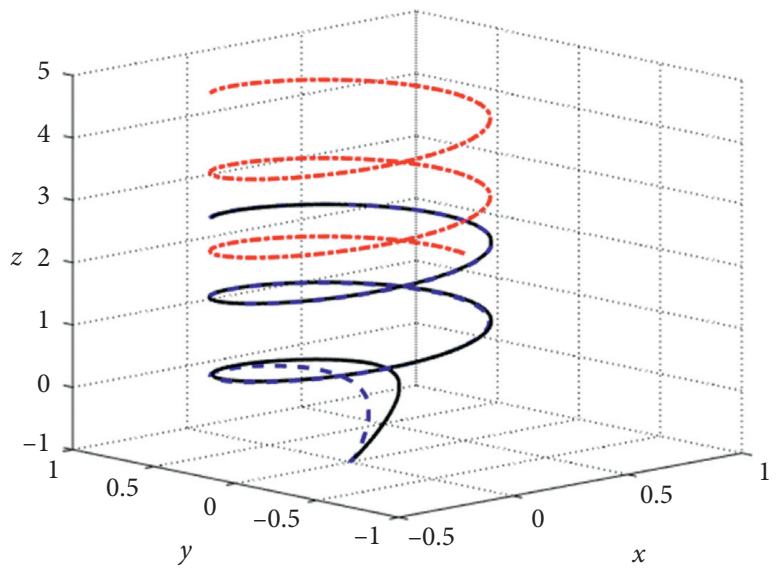

... - Target trajectory

- PC \& STSM tracking

- - - 2-SMC tracking

Figure 7: Continued. 

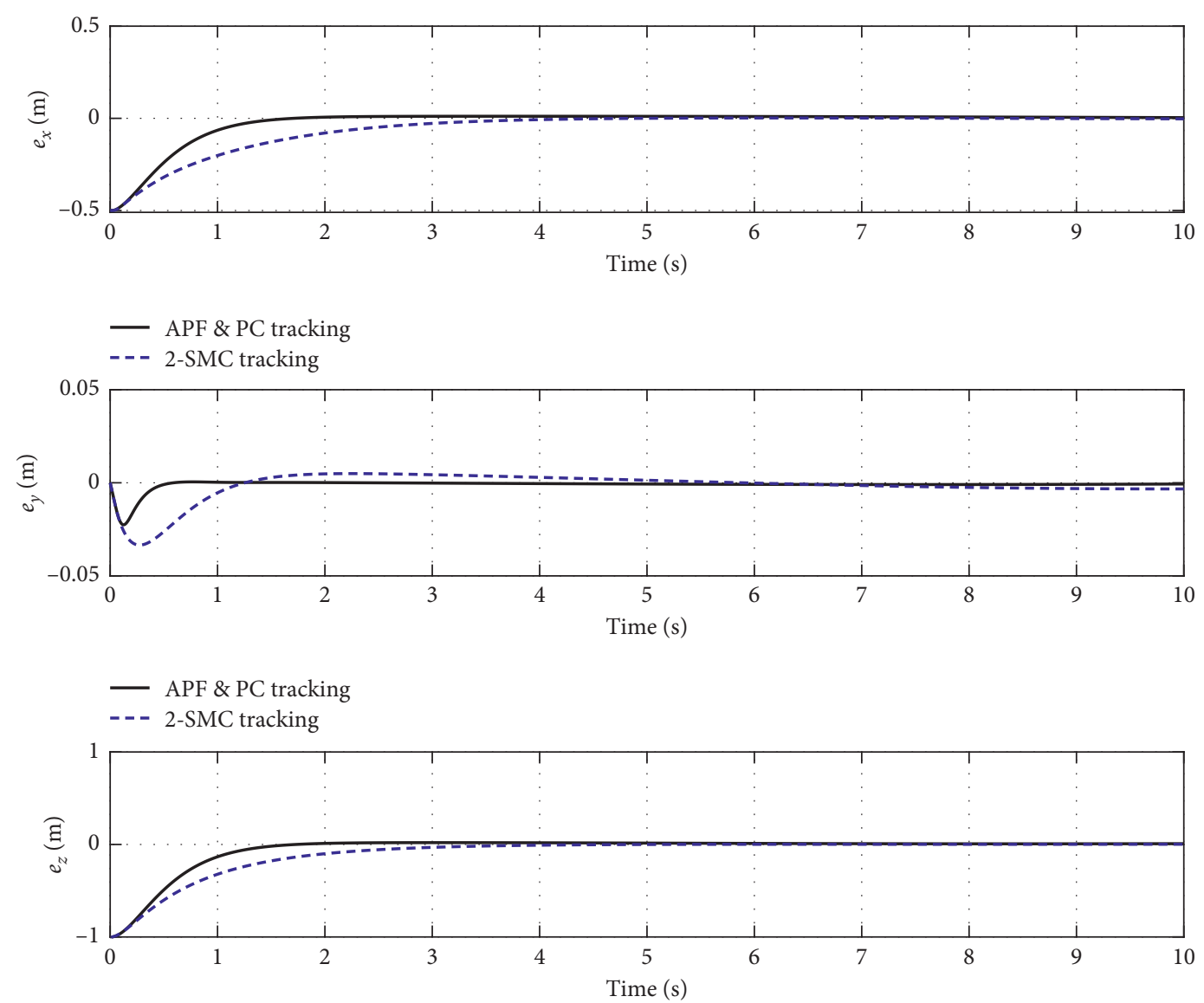

- APF \& PC tracking

-- - 2-SMC tracking

(b)

Figure 7: Continued. 

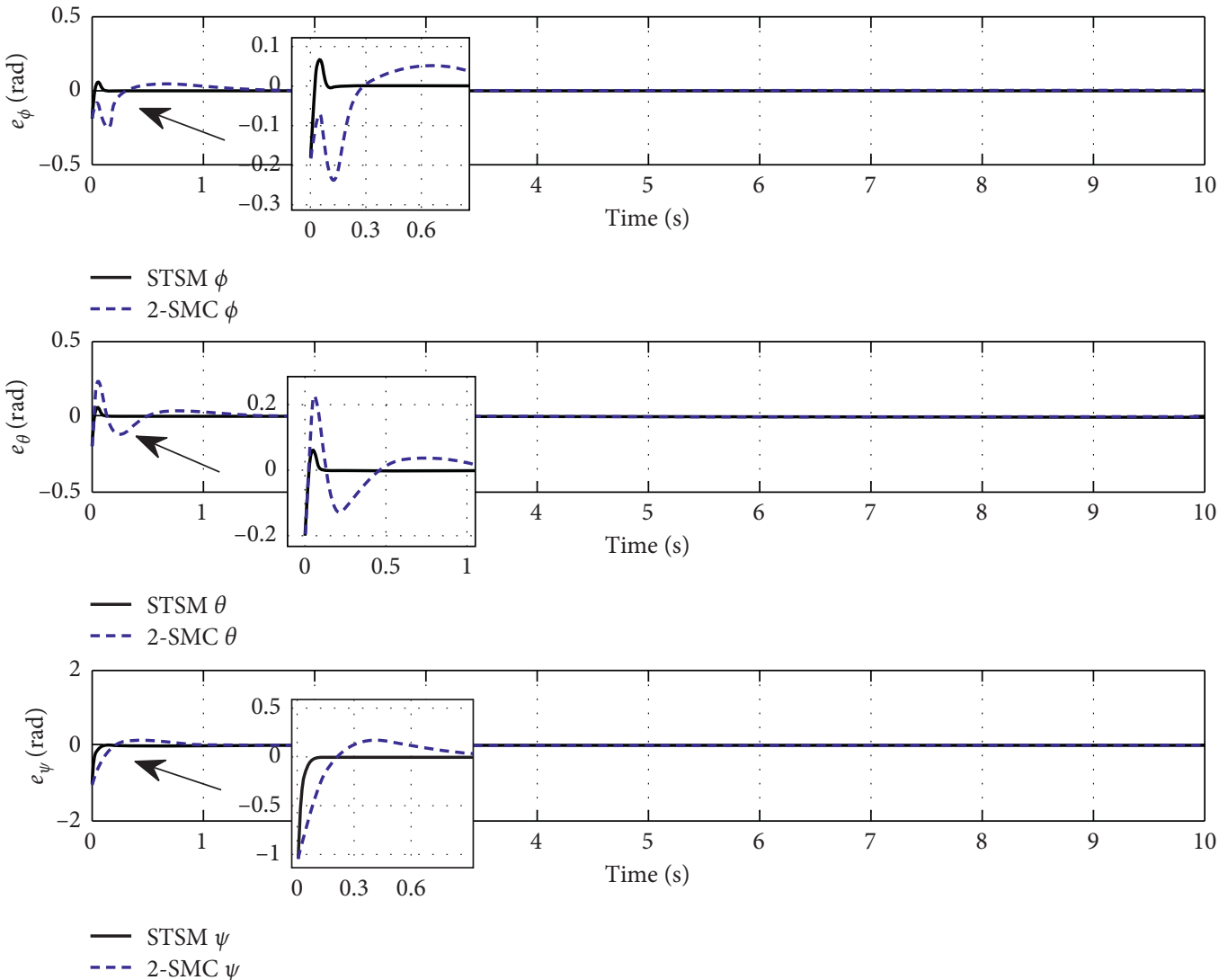

(c)

Figure 7: Aerial moving target tracking. (a) Trajectory tracking. (b) Position tracking error. (c) Attitude tracking error.

TABLE 3: Convergence time of tracking error.

\begin{tabular}{lcccccc}
\hline Method & $x(\mathrm{~s})$ & $y(\mathrm{~s})$ & $z(\mathrm{~s})$ & $\phi(\mathrm{s})$ & $\theta(\mathrm{s})$ & \\
\hline PC\&STSM & 1.6 & 1.4 & 1.6 & 0.35 & 0.35 & 0.25 \\
2-SMC & 3.6 & 4.2 & 3.8 & 1.5 & 1.4 & 1.2 \\
\hline
\end{tabular}

TABLE 4: Overshoot of tracking error.

\begin{tabular}{lllllll}
\hline Method & $x$ & $y$ & $z$ & $\phi$ & $\theta$ & \\
\hline PC\&STSM & 0 & 0 & 0 & 0.09 & 0.08 & 0 \\
2-SMC & 0 & 0.05 & 0 & 0.05 & 0.24 & 0.18 \\
\hline
\end{tabular}




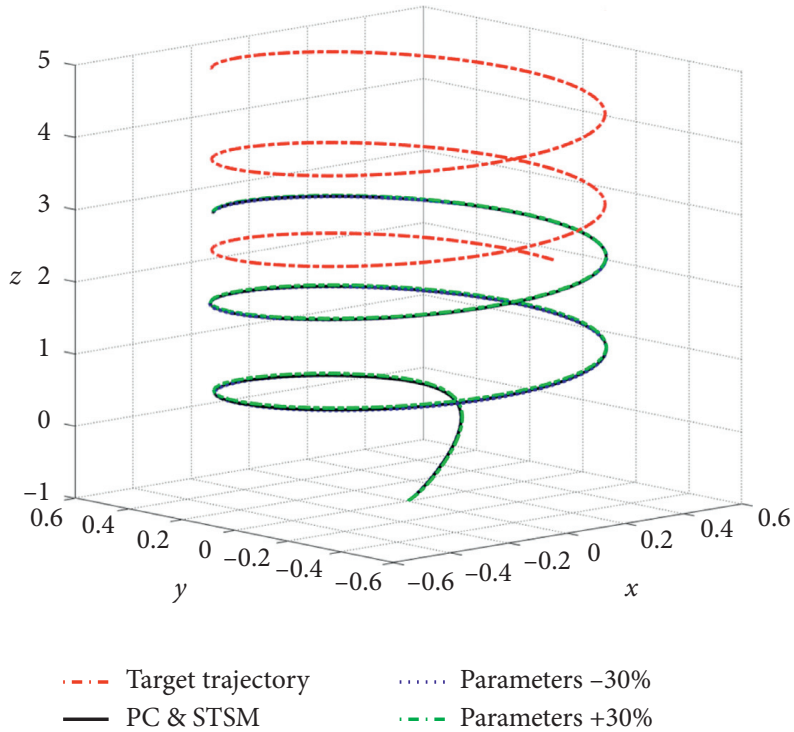

(a)

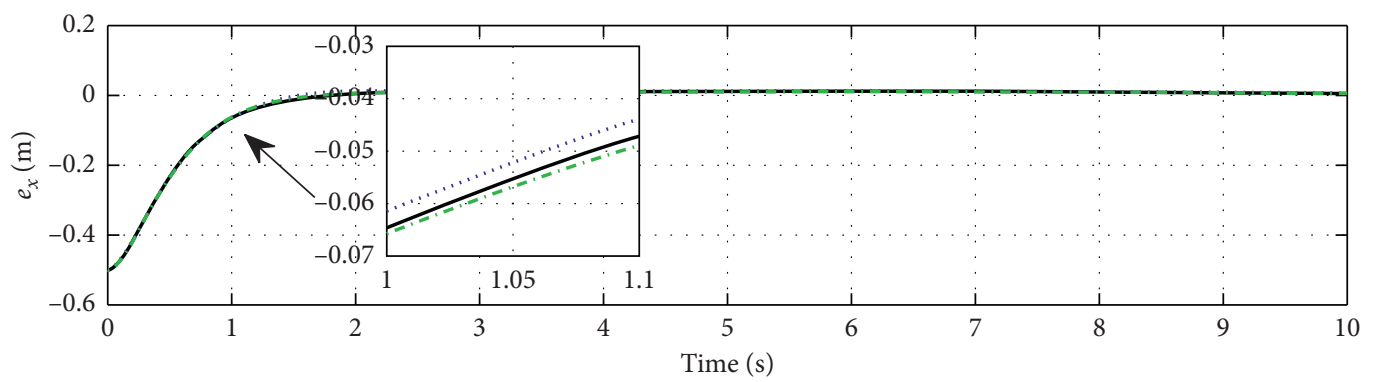

— APF \& PC tracking

.... Parameters $-30 \%$

- - Parameters $+30 \%$

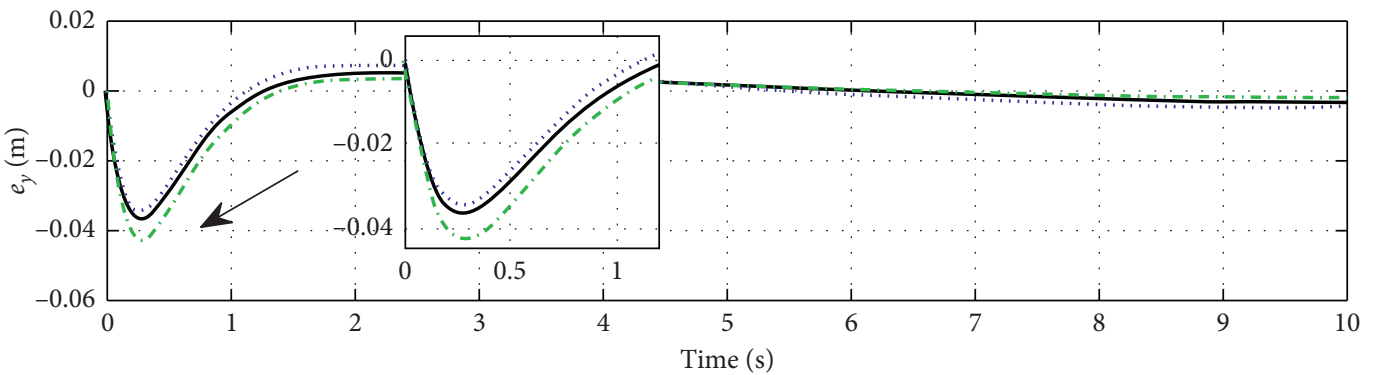

- APF \& PC tracking

..... Parameters $-30 \%$

- - Parameters $+30 \%$

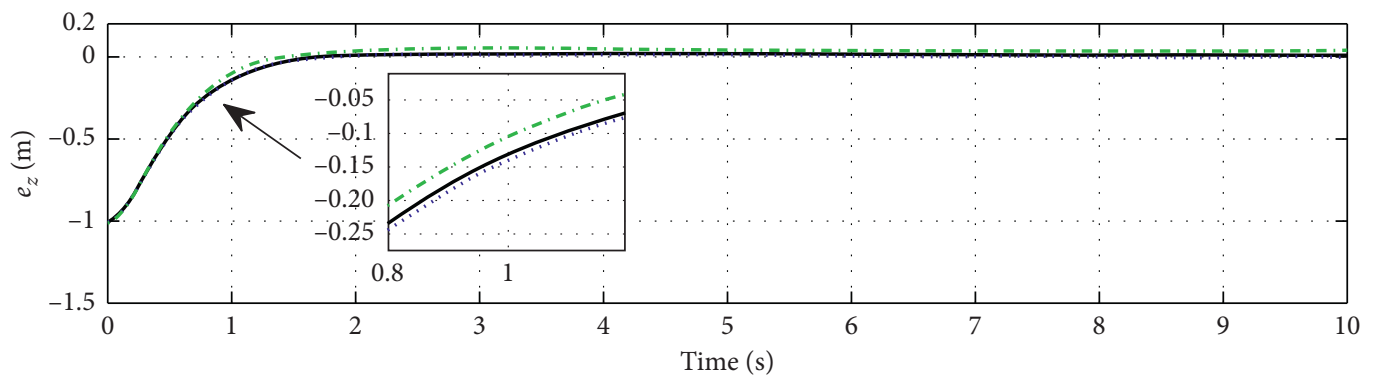

— APF \& PC tracking

.... Parameters $-30 \%$

- - Parameters $+30 \%$

(b)

Figure 8: Continued. 

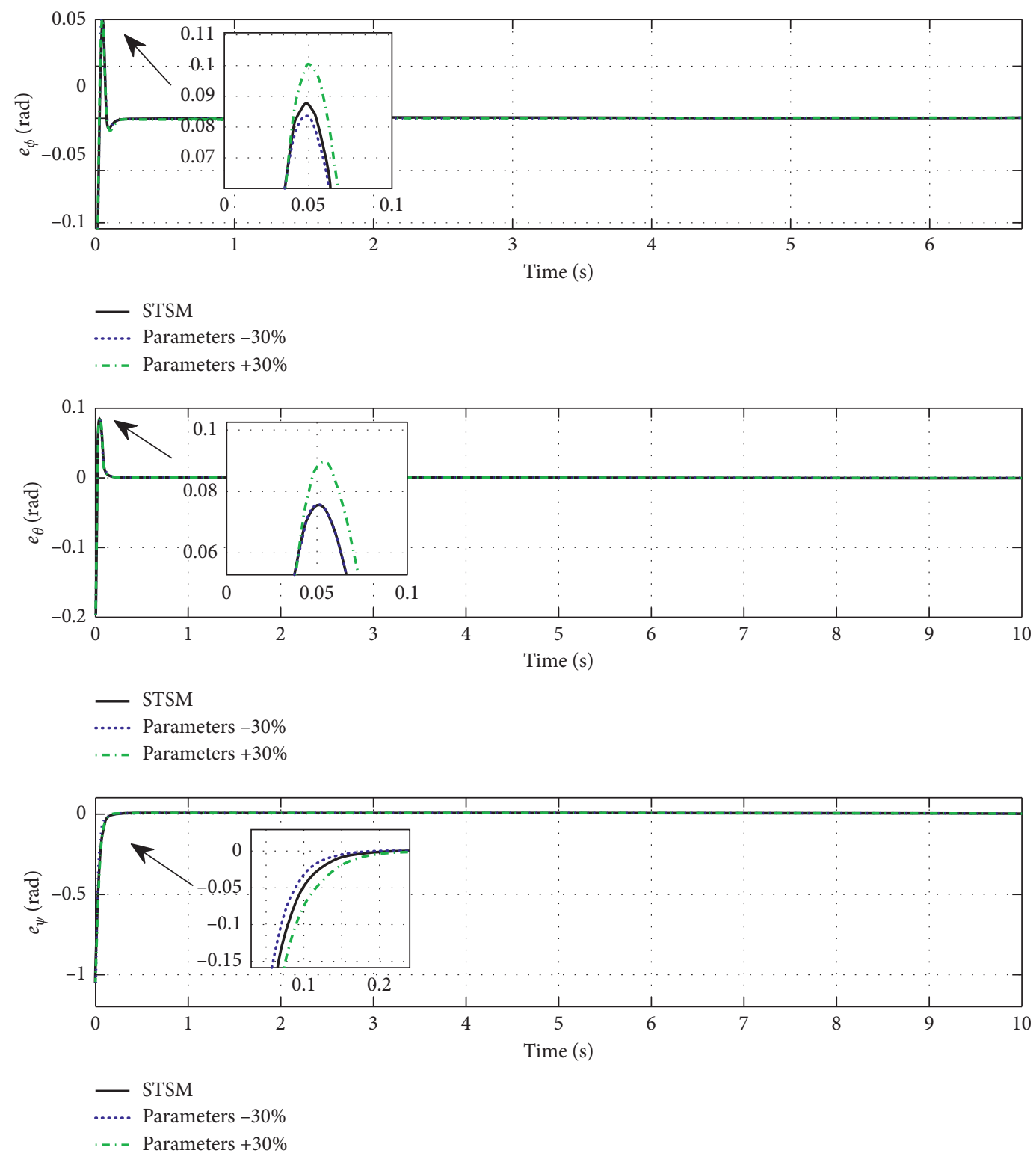

(c)

Figure 8: Target tracking with uncertainties and disturbances. (a) Trajectory tracking. (b) Position tracking error. (c) Attitude tracking error.

\section{Conclusion}

In this paper, we propose a composite control strategy based on passive control and super-twisting sliding mode control to solve the problem of moving target tracking of quadrotors considering model uncertainties and external disturbances. The control structure is composed of position and an attitude controller. For position control, the artificial potential field method is proposed by combining gravitational potential with repulsion potential to achieve the stable tracking of moving targets at a fixed distance. Meanwhile, the cascade passive control is designed to ensure the stability of the positioning system. Moreover, the super-twisting sliding mode controller with an improved reaching law is introduced to make the attitude angles quickly track the desired trajectory and overcome uncertainties and disturbances. Finally, the simulation results show that the proposed control system can achieve a better tracking performance and robustness compared with 2-SMC in moving target tracking.

\section{Data Availability}

No data were used to support this study.

\section{Conflicts of Interest}

The authors declare that they have no conflicts of interest. 


\section{Acknowledgments}

This work was supported in part by the National Natural Science Foundation of China under Grant 61903163, Natural Science Foundation of the Jiangsu Higher Education Institutions of China under Grant 19KJB510023, and Marine Equipment and Technology Institute of JUST under Grant HZ20200007.

\section{References}

[1] B. Liu, W. Zhang, W. Chen, H. Huang, and S. Guo, "Online computation offloading and traffic routing for UAV swarms in edge-cloud computing," IEEE Transactions on Vehicular Technology, vol. 69, no. 8, pp. 8777-8791, 2020.

[2] S. S. Esfahlan, "Mixed reality and remote sensing application of unmanned aerial vehicle in fire and smoke detection," Journal of Industrial Information Integration, vol. 15, pp. 42-49, 2019.

[3] S. Yang, J. Han, L. Xia, and Y.-H. Chen, "Adaptive robust servo constraint tracking control for an underactuated quadrotor UAV with mismatched uncertainties," ISA Transactions, vol. 106, pp. 12-30, 2020.

[4] H. Ye, X. Yang, H. Shen, and R. Li, "Standoff tracking of a moving target for quadrotor using Lyapunov potential function," International Journal of Control, Automation and Systems, vol. 18, pp. 845-855, 2020.

[5] S. Mario, T. Andrea, E. Zenerino, and M. Chiaberge, "Multipurpose UAV for search and rescue operations in mountain avalanche events," Geomatics, Natural Hazards and Risk, vol. 8, pp. 18-33, 2017.

[6] O. Tekinalp, .A. K Ali, D. Kaya et al., "Propulsion system selection and modeling for a quadrotor with search and rescue mission," in Proceedings of the 54th AIAA Aerospace Sciences Meeting, San Diego, CA, USA, January 2016.

[7] S. Wang, F. Jiang, B. Zhang, R. Ma, and Q. Hao, "Development of UAV-based target tracking and recognition systems," IEEE Transactions on Intelligent Transportation Systems, vol. 21, no. 8, pp. 3409-3422, 2020.

[8] M. Rabah, A. Rohan, S. A. S. Mohamed, and S.-H. Kim, "Autonomous moving target-tracking for a UAV quadcopter based on fuzzy-PI," IEEE Access, vol. 7, pp. 38407-38419, 2019.

[9] O. Araar, N. Aouf, and I. Vitanov, "Vision based autonomous landing of multirotor UAV on moving platform," Journal of Intelligent \& Robotic Systems, vol. 85, no. 2, pp. 369-384, 2017.

[10] X. Chen, W. Xue, H. Qiu, and H. Ye, "A moving target tracking control and obstacle avoidance of quadrotor UAV based on sliding mode control using artificial potential field and RBF neural networks," in Proceedings of the 39th Chinese Control Conference, Shenyang China, July 2020.

[11] M. C. Roger and L. T. Aguilar, "Robust PID control of quadrotors with power reduction analysis," ISA Transactions, vol. 98, pp. 47-62, 2020.

[12] Y. Zhang, Z. Chen, X. Zhang, Q. Sun, and M. Sun, “A novel control scheme for quadrotor UAV based upon active disturbance rejection control," Aerospace Science and Technology, vol. 79, pp. 601-609, 2018.

[13] L. Martins, C. Cardeira, and P. Oliveira, "Feedback linearization with zero dynamics stabilization for quadrotor control," Journal of Intelligent \& Robotic Systems, vol. 101, no. 7, 2021.
[14] N. Koksal, H. An, and B. Fidan, "Backstepping-based adaptive control of a quadrotor UAV with guaranteed tracking performance," ISA Transactions, vol. 105, pp. 98-110, 2020.

[15] A. Eskandarpour and I. Sharf, "A constrained error-based MPC for path following of quadrotor with stability analysis," Nonlinear Dynamics, vol. 99, no. 2, pp. 899-918, 2020.

[16] S. Yang and B. Xian, "Energy-based nonlinear adaptive control design for the quadrotor UAV system with a suspended payload," IEEE Transactions on Industrial Electronics, vol. 67, no. 3, pp. 2054-2064, 2020.

[17] Y. Zou and Z. Meng, "Immersion and invariance-based adaptive controller for quadrotor systems," IEEE Transactions on Systems, Man, and Cybernetics: Systems, vol. 49, no. 11, pp. 2288-2297, 2019.

[18] X. Lin, J. Liu, Y. Yu, and C. Sun, "Event-triggered reinforcement learning control for the quadrotor UAV with actuator saturation," Neurocomputing, vol. 415, no. 11, pp. 135-145, 2020.

[19] J. M. Vazquez-Nicolas, E. Zamora, I. González-Hernández, R. Lozano, and H. Sossa, "PD+SMC quadrotor control for altitude and crack Recognition using deep learning," International Journal of Control, Automation and Systems, vol. 18, no. 5, pp. 834-844, 2020.

[20] S. Shao, M. Chen, J. Hou, and Q. Zhao, "Event-triggeredbased discrete-time neural control for a quadrotor UAV using disturbance observer," IEEE/ASME Transactions on Mechatronics, vol. 26, no. 2, pp. 689-699, 2021.

[21] K. Mei, L. Ma, R. He, and S. Ding, "Finite-time controller design of multiple integrator nonlinear systems with input saturation," Applied Mathematics and Computation, vol. 372, Article ID 124986, 2020.

[22] L. Liu, W. X. Zheng, and S. Ding, "An adaptive SOSM controller design by using a sliding-mode-based filter and its application to buck converter," IEEE Transactions on Circuits and Systems I: Regular Papers, vol. 67, no. 7, pp. 2409-2418, 2020.

[23] S. Ding, W.-H. Chen, K. Mei, and D. J. Murray-Smith, "Disturbance observer design for nonlinear systems represented by input-output models," IEEE Transactions on Industrial Electronics, vol. 67, no. 2, pp. 1222-1232, 2020.

[24] F. Chen, R. Jiang, K. Zhang, B. Jiang, and G. Tao, "Robust backstepping sliding-mode control and observer-based fault estimation for a quadrotor UAV," IEEE Transactions on Industrial Electronics, vol. 63, no. 8, pp. 5044-5056, 2016.

[25] G. Xu, Y. Xia, D. H. Zhai, and D. Ma, "Adaptive prescribed performance terminal sliding mode attitude control for quadrotor under input saturation," IET Control Theory \& Applications, vol. 14, no. 17, pp. 2473-2480, 2020.

[26] H. Castañeda, O. S. Salas-Peña, and J. D. León-Morales, "Extended observer based on adaptive second order sliding mode control for a fixed wing UAV," ISA Transactions, vol. 66, pp. 226-232, 2017.

[27] Y. Du, X. Zhang, and Z. Nie, "A real-time collision avoidance strategy in dynamic airspace based on dynamic artificial potential field algorithm," IEEE Access, vol. 7, pp. 169469169479, 2019.

[28] Y.-B. Chen, G.-C. Luo, Y.-S. Mei, J.-Q. Yu, and X.-L. Su, "UAV path planning using artificial potential field method updated by optimal control theory," International Journal of Systems Science, vol. 47, no. 6, pp. 1407-1420, 2016.

[29] Q. Hu, H. Dong, Y. Zhang, and G. Ma, "Tracking control of spacecraft formation flying with collision avoidance," Aerospace Science and Technology, vol. 42, pp. 353-364, 2015. 
[30] R. Ortega, A. van der Schaft, F. Castanos, and A. Astolfi, "Control by interconnection and standard passivity-based control of port-hamiltonian systems," IEEE Transactions on Automatic Control, vol. 53, no. 11, pp. 2527-2542, 2008.

[31] C. Ha, Z. Zuo, F. B. Choi, and D. Lee, "Passivity-based adaptive backstepping control of quadrotor-type UAVs," Robotics and Autonomous Systems, vol. 62, no. 9, pp. 13051315, 2014.

[32] G. R. Jose and R. Hugo, "Asymptotic stability for a transformed nonlinear UAV model with a suspended load via energy shaping," European Journal of Control, vol. 52, pp. 87-96, 2020.

[33] G. V. Raffo, M. G. Ortega, and F. R. Rubio, "An integral predictive/nonlinear $\mathrm{H} \infty$ control structure for a quadrotor helicopter," Automatica, vol. 46, no. 1, pp. 29-39, 2010.

[34] A. Das, F. Lewis, and K. Subbarao, "Backstepping approach for controlling a quadrotor using Lagrange form dynamics," Journal of Intelligent and Robotic Systems, vol. 56, no. 1-2, pp. 127-151, 2009.

[35] N. Bu, M. Deng, and M. Deng, "Passivity-based tracking control for uncertain nonlinear feedback systems," Journal of Robotics and Mechatronics, vol. 28, no. 6, pp. 837-841, 2016.

[36] E.-H. Zheng, J.-J. Xiong, and J.-L. Luo, "Second order sliding mode control for a quadrotor UAV," ISA Transactions, vol. 53, no. 4, pp. 1350-1356, 2014. 